Linköping Studies in Science and Technology

Thesis No. 1791

\title{
Non-linear inverse geothermal problems
}

\author{
Dennis Wokiyi
}

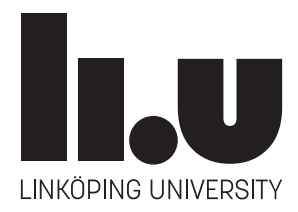

Department of Mathematics

Division of Mathematics and Applied Mathematics

Linköping University, SE-581 83 Linköping, Sweden

Linköping 2017 
Linköping Studies in Science and Technology. Thesis No. 1791 Licentiate Thesis

(C) 2017 Dennis Wokiyi

Division of Mathematics and Applied Mathematics

Department of Mathematics

Linköping University

SE-581 83, Linköping, Sweden

dennis.wokiyi@liu.se

www.mai.liu.se

Typeset by the author in $\mathrm{IAT}_{\mathrm{E}} \mathrm{X} 2 \mathrm{e}$ documentation system.

ISSN 0280-7971

ISBN 978-91-7685-404-4

Printed by LiU-Tryck, Linköping, Sweden 2017 


\section{Abstract}

The inverse geothermal problem consist of estimating the temperature distribution below the earth's surface using temperature and heat-flux measurements on the earth's surface. The problem is important since temperature governs a variety of the geological processes including formation of magmas, minerals, fosil fuels and also deformation of rocks. Mathematical this problem is formulated as a Cauchy problem for an non-linear elliptic equation and since the thermal properties of the rocks depend strongly on the temperature, the problem is non-linear. This problem is ill-posed in the sense that it does not satisfy atleast one of Hadamard's definition of well-posedness.

We formulated the problem as an ill-posed non-linear operator equation which is defined in terms of solving a well-posed boundary problem. We demonstrate existence of a unique solution to this well-posed problem and give stability estimates in appropriate function spaces. We show that the operator equation is well-defined in appropriate function spaces.

Since the problem is ill-posed, regularization is needed to stabilize computations. We demostrate that Tikhonov regularization can be implemented efficiently for solving the operator equation. The algorithm is based on having a code for solving a wellposed problem related to the operator equation. In this study we demostrate that the algorithm works efficiently for $2 D$ calculations but can also be modified to work for $3 \mathrm{D}$ calculations. 



\section{Acknowledgments}

I take this opportunity to express my gratitude to my supervisors Vladmir Kozlov and Fredrik Berntsson for the guidance, patience and good collaboration during my studies at Linköping University. In the same way I wish to thank the entire community at the Department of Mathematics, Linköping University for the good working environment and support.

My studies at Linköping University would not have been possible without the support of the Department of Mathematics, Makerere University. I thank my supervisors, Godwin Kakuba and John M.Mango whom I work with when at Makerere University for all the support offered to me during my studies.

To my family, there is no measure of words that can express my gratefulness to your support. A special thank you to my parents Joseph Mwigale Wasagami and Loyce Namataka for all the sacrifices you have taken to support me this far.

I would like to thank ISP and the Eastern Africa Universities mathematics Programme (EAUMP) for the financial support. Special thank you to the coordinators; Leif Abrahamson, John Mango, Juma Kasozi and other staff at ISP for always ensuring that my stay in Sweden is comfortable. 


\section{Contents}

1 Introduction 1

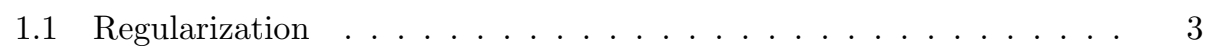

1.1.1 Compact operators . . . . . . . . . . . . . . 7

1.1.2 Tikhonov Regularization . . . . . . . . . . . . . . . . . . 9

1.1.3 Parameter Choice strategies . . . . . . . . . . . . . . 11

1.1.4 Concrete example . . . . . . . . . . . . . 12

2 Summary of Papers $\quad 17$

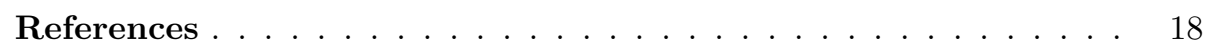

\section{INCLUDED PAPERS}

I. An efficient regularization method for a large scale ill-posed geothermal problem

II. Solvability of an inverse non-linear geothermal problem 


\section{Introduction}

Inverse problems arise in many industrial, engineering and biomedical applications. Many of the inverse problems that arise in these fields can mathematically be formulated as Cauchy problems for elliptic partial differential equations; examples include a classical thermostatics problem which consists of recovering the temperature in a given domain when it's distribution and heat flux are known over the accessible region of the boundary $[23,5,4,25]$, electrostatics problem encountered in electric impedance tomography $[13,12]$, corrosion detection $[18,17,1]$, inverse scattering problems $[11,22,3]$. Cauchy problems for elliptic equations as with many other inverse problems are known to be ill-posed.

According to Hadamard's definition of well-posedness [14, 16, 19, 10, 27], a problem is said to be well-posed if it has the following properties

1. There exists a solution of the problem (existence).

2. There is at most one solution of the problem (uniqueness).

3. The solution depends continuously on the data (stability).

Problems that fail to meet these requirements are said to be ill-posed. This is not a precise mathematical definition; to make it precise in an actual application, one has to specify the notion of a solution, which data are considered admissible, and which topology is used to measuring continuity. Mathematically, existence of a solution can be enforced by enlarging the solution space. If a problem has more than one solution, then information about the model is missing and in this case, additional properties can be built into the model. The requirement that the solution depends continuously on the available data is the most important. If a problem lacks the 
property of stability, then it's solution is practically impossible to compute because any measurements or numerical computation are polluted by unavoidable errors: thus the data of the problem are always perturbed by noise. If the solution of the problem does not depend continuously on the data, then in general the computed solution has nothing to do with the true solution. Indeed there is no way to overcome this difficulty unless additional information about the solution is available.

For examples of ill-posed problems, we consider two Cauchy problems, problems for which data is only defined on a part of a boundary, that is Cauchy problem for Laplace, a classical example given by Hadamard in his famous example and the Cauchy problem for the Helmholtz equation.

Example 1 Consider problem

$$
\Delta u=0 \text { in } \Omega=(0,1) \times(0,1)
$$

with initial conditions

$$
u(x, 0)=f(x), \quad u_{y}(x, 0)=g(x), \quad x \in(0,1),
$$

where the functions $f$ and $g$ are given Cauchy data. The unique solution for $f(x)=0$ and $g(x)=\frac{1}{n \pi} \sin (n \pi x)$ is given by

$$
u(x, y)=\frac{\sinh (n \pi x) \sin (n \pi x)}{n^{2} \pi^{2}} .
$$

It is easy to see that there is no continuous dependence on available data, since $u_{y} \rightarrow 0$ as $n \rightarrow \infty$ but $u(x, y) \rightarrow \infty$ (for $\left.y>0\right)$ and hence the problem is ill-posed. It is also well known that this problem can be stabilized by adding the condition requiring that $\|u\| \leq M[9,7,8]$.

Example 2 Consider the Cauchy problem for a Helmholtz equation

$$
\begin{cases}\Delta u(x, y)+k^{2} u(x, y)=0, & \text { in }(0, a) \times(0, b), \\ u(x, 0)=f(x), u_{y}(x, 0)=g(x), & \text { for all } x \in[0, a], \\ u(0, y)=u(a, y)=0 & \text { for all } y \in[0, b],\end{cases}
$$

where $k$ is a the wave number, $f, g \in L^{2}(0, a)$ are specified Cauchy data. By separation of variables the solution to this problem is given by

$$
u(x, y)=\sum_{n=1}^{\infty} \sin \frac{n \pi x}{a}\left(A_{n} \cosh \lambda_{n} y+\lambda_{n}^{-1} B_{n} \sinh \lambda_{n} y\right), \quad \lambda_{n}=\sqrt{a^{-2} n^{2} \pi^{2}-k^{2}},
$$


where the coefficients $A_{n}$ and $B_{n}$ are given by

$$
A_{n}=\int_{0}^{a} f(x) \sin \frac{n \pi x}{a} d x \text { and } B_{n}=\int_{0}^{a} g(x) \sin \frac{n \pi x}{a} d x
$$

For initial data $f(x)=\frac{\sin (n \pi x)}{n^{2} k^{2}}$ and $g(x)=0$, the exact solution is given by

$$
u_{n}(x, y)=\frac{a \sin \left(\frac{n \pi x}{a}\right) \cosh \left(\lambda_{n} y\right)}{2 n^{2} k^{2}} .
$$

We find that $\sup _{x \in[0, a]}\{|f(x)|+|g(x)|\} \rightarrow 0$ but $\sup _{x \in[0, a]} \| u(x, y) \mid \rightarrow \infty$ as $n \rightarrow \infty$ for fixed $y>0$. Therefore the estimate $\|u\|_{\infty} \leq C\left(\|f\|_{\infty}+\|g\|_{\infty}\right)$ can not hold in general and the requirement that the solution depends continuously on the data does not hold and therefore the problem is ill-posed [21].

Violation of the stability property creates serious numerical problems and if one is to approximate a problem whose solution does not depend on the data continuously by traditional numerical methods, as one would use for well-posed problems, then one expects that the numerical methods become unstable. Therefore to approximate solutions to problems that violate the stability property, one uses regularization methods. Regularization methods consist of reformulating the problem so that the solution to the regularized problem is less sensitive to perturbations.

\section{$1.1 \quad$ Regularization}

Many inverse problems can be formulated as operator equations of the form

$$
K x=y,
$$

where $K$ is a bounded linear compact operator between Hilbert spaces $X$ and $Y$. For simplicity, it is assumed that the compact operator $K$ is one-to-one. This is not too restrictive because we can always replace the domain $X$ with the orthogonal complement of the kernel of $K$. We also make the assumption that there exists a solution $x \in X$ of the unperturbed equation $K x=y$. In other words it is assumed that $y \in \mathcal{R}(K)$. The injectivity of $K$ implies that this solution is unique. It would be too restrictive to assume that the range of $K$ is a closed subset of $Y$, since in that case $K^{-1}$ is also bounded. 
In a real application, the right-hand side $y \in Y$ is never known exactly but only upto an error of, say $\delta>0$. Therefore, we assume that we know $y^{\delta} \in Y, \delta>0$ such that

$$
\left\|y-y^{\delta}\right\| \leq \delta
$$

The aim therefore is to solve the perturbed equation

$$
K x^{\delta}=y^{\delta}
$$

This problem is usually not solvable because we can not assume that the measured data $y^{\delta}$ is in the range $\mathcal{R}(K)$ of $K$. Therefore the best we can do is to determine an approximation $x_{\alpha}^{\delta} \in X$ of the exact solution $x$. The approximate solution $x_{\alpha}^{\delta}$ should depend continuously on the available data $y^{\delta}$ so that it can be computed in a stable way and should also have the property that as the noise level $\delta$ decreases to zero and the parameter $\alpha$ is chosen appropriately, then $x_{\alpha}^{\delta}$ tends to $x$. It is therefore the goal of regularization methods to construct a suitable bounded approximation $R_{\alpha}: Y \rightarrow X$ of the unbounded inverse operator $K^{-1}: \mathcal{R}(K) \rightarrow X$, see [10]. The following definition can be found in [19].

Definition 1.1.1. A regularization strategy is a family of linear and bounded operators

$$
R_{\alpha}: Y \rightarrow X, \quad \alpha>0
$$

such that

$$
\lim _{\alpha \rightarrow 0} R_{\alpha} K x=x, \forall x \in X
$$

The positive parameter $\alpha$ is called the regularization parameter.

The choice of the regularization parameter $\alpha$ should be in such away that as the noise level $\delta$ tends to zero, the regularized solution $x_{\alpha}^{\delta}$ should tend to the exact solution $x$. Therefore, the parameter $\alpha$ should somehow be dependent on the noise level $\delta>0, y^{\delta}$ and possibly other information about the operator $K$, or about $y$.

The notion of a regularization strategy is based on unpertubed data; that is the regularizer $R_{\alpha} y$ converges to $x$ for the exact righthand side $y=K x$. Let $y \in \mathcal{R}(K)$ be the exact righthand side, $y^{\delta}$ be the measured data with $\left\|y^{\delta}-y\right\| \leq \delta$ and $x_{\alpha}^{\delta}:=R_{\alpha} y^{\delta}$ be an approximation of the solution $x$ of $K x=y$. The error between the computed 
solution and the exact solution is split into two parts

$$
\begin{aligned}
\left\|x_{\alpha}^{\delta}-x\right\| & \leq\left\|R_{\alpha} y^{\delta}-R_{\alpha} y\right\|+\left\|R_{\alpha} y-x\right\| \leq\left\|R_{\alpha}\right\|\left\|y^{\delta}-y\right\|+\left\|R_{\alpha} K x-x\right\| \\
& \leq \delta\left\|R_{\alpha}\right\|+\left\|R_{\alpha} K x-x\right\| .
\end{aligned}
$$

We observe that the first term in the total error is the error in the measured data multiplied by the number $\left\|R_{\alpha}\right\|$ of the regularized problem and the second term is simply the estimation error. If the operator $R_{\alpha}$ is uniformly bounded, then it is clear to see that $\delta\left\|R_{\alpha}\right\|$ tends to 0 as $\delta \rightarrow 0$. Similarly by definition of a regularization strategy the estimation error $\left\|R_{\alpha} K x-x\right\|$ for exact data $y$ tends to zero as $\alpha \rightarrow 0$. On the otherhand if the operators $R_{\alpha}$ are not uniformly bounded; that is there exists a sequence $\left\{\alpha_{j}\right\}$ such that $\left\|R_{\alpha_{j}}\right\| \rightarrow \infty$ for $j \rightarrow \infty$, then the term $\delta\left\|R_{\alpha}\right\|$ tends to infinity as $\alpha \rightarrow 0$; see Theorem 2.2 [19]. Therefore to keep the total error as small as possible, the strategy is to choose the parameter $\alpha=\alpha(\delta)$ which is dependent on $\delta[10,19]$.

This discussion leads to the following definition of a regularization method taken from $[10]$

Definition 1.1.2. Let $K: X \rightarrow Y$ be a bounded linear operator between the Hilbert spaces $X$ and $Y, \alpha_{0} \in(0,+\infty]$. For every $\alpha \in\left(0, \alpha_{0}\right)$, let

$$
R_{\alpha}: Y \rightarrow X
$$

be continuous(not necessary linear) operator. The family $\left\{R_{\alpha}\right\}$ is called a regularization or a regularization operator for $K^{-1}$, if for all $y \in \mathcal{D}\left(K^{-1}\right)$, there exists a parameter choice rule $\alpha=\alpha\left(\delta, y^{\delta}\right)$ such that

$$
\limsup _{\delta \rightarrow}\left\{\left\|R_{\alpha\left(\delta, y^{\delta}\right)} y^{\delta}-K^{-1} y\right\| \mid y^{\delta} \in Y,\left\|y^{\delta}-y\right\| \leq \delta\right\}=0
$$

holds. Here $\alpha: \mathbb{R}^{+} \times Y \rightarrow(0, \alpha)$ is such that

$$
\lim _{\delta \rightarrow 0} \sup \left\{\alpha\left(\delta, y^{\delta}\right) \mid y^{\delta} \in Y,\left\|y^{\delta}-y\right\| \leq \delta\right\}=0 .
$$

For a specific $y \in \mathcal{D}\left(K^{-1}\right)$, The pair $\left(\alpha, R_{\alpha}\right)$ is called a (convergent) regularization method for solving $y=K x$ if (1.13) and (1.14) hold.

\section{Example}


Numerical differentiation has properties of an ill-posed problem and since many inverse problems involve, at some step, differentiation of data, differentiation can be viewed as an inverse problem. Consider a function $f \in C^{1}[0,1], \delta \in(0,1)$ and $n \in \mathbb{N}$, with $n \geq 2$. Define

$$
f_{n}^{\delta}(x)=f(x)+\delta \sin \frac{n x}{\delta}, \quad x \in[0,1],
$$

where $f$ and $f_{n}^{\delta}$ are the exact and perturbed data respectively, The function $f_{n}^{\delta}$ has a derivative

$$
\left(f_{n}^{\delta}\right)^{\prime}=f^{\prime}(x)+n \cos \frac{n x}{\delta}, \quad x \in[0,1] .
$$

We observe that $\left\|f_{n}^{\delta}-f\right\|_{\infty}=\delta$ and $\left\|\left(f_{n}^{\delta}\right)^{\prime}-f^{\prime}\right\|_{\infty}=n$, meaning for an arbitrarily small error $\delta$ in the data, the error in the derivative can be arbitrarily large as $n \rightarrow \infty$. Hence numerical differentiation does not depend continuously on the data with respect to the maximum norm. One way to overcome the violation of the stability property is to use mollification for regularizing numerical differentiation. To this end we first introduce

$$
\rho_{\varepsilon}(x)=\frac{1}{\varepsilon \sqrt{\pi}} e^{-\left(\frac{x}{\varepsilon}\right)^{2}}
$$

the Gaussian kernel with blurring radius $\varepsilon$. We observe that $\rho_{\varepsilon}$ is an infinitely differentiable function that falls to nearly 0 outside a few radii from it's center $\approx 3 \varepsilon$, is positive and has integral 1. After extending the exact data function $f$ and the measured data function $f^{\delta}$ to the interval $I_{\varepsilon}=[-3 \varepsilon, 1+3 \varepsilon]$ in such away that they decay smoothly to zero in $[-3 \varepsilon, 0] \cup[1,1+3 \varepsilon]$ and they are zero in $\mathbb{R}-I_{\varepsilon}$. That is the extensions can be acomplished for instance by defining

$$
\begin{aligned}
& f^{\delta}(x)=f^{\delta}(0) \exp \left\{x^{2} /\left[(3 \varepsilon)^{2}-x^{2}\right]\right\}, \quad-3 \varepsilon \leq x \leq 0, \\
& f^{\delta}(x)=f^{\delta}(1) \exp \left\{(x-1)^{2} /\left[(3 \varepsilon)^{2}-(x-1)^{2}\right]\right\}, \quad 1 \leq x \leq 1+3 \varepsilon .
\end{aligned}
$$

The convolution

$$
J_{\varepsilon} f=\left(\rho_{\varepsilon} * f\right)(x)=\int_{\infty}^{\infty} \rho_{\varepsilon}(x-s) f(s) d s,
$$

defines a $C^{\infty}$ function in the entire line. $J_{\varepsilon} f$ is the mollifier of $f$ and $\varepsilon$ is the radius of mollification. From this definition, it imediately follows that

$$
\frac{d}{d x} J_{\varepsilon} f=\left(\rho_{\varepsilon} * f\right)^{\prime}(x)=\left(\rho_{\varepsilon}^{\prime} * f\right)(x) .
$$

The following results can be found in [6]. 
Lemma 1.1.1. (Consistency) If $\left\|f^{\prime \prime}\right\|_{\infty, I} \leq M$, where $M$ is a constant, then

$$
\left\|\left(J_{\varepsilon} f\right)^{\prime}-f^{\prime}\right\|_{\infty, I} \leq 3 \varepsilon M
$$

Lemma 1.1.2. (Stability) If $f^{\delta}(x) \in C^{0}(I)$ and $\left\|f^{\delta}-f\right\|_{\infty, I} \leq \delta$, then

$$
\|\left(\left(J_{\varepsilon} f^{\delta}\right)^{\prime}-\left(J_{\varepsilon} f\right)^{\prime} \|_{\infty, I} \leq \frac{2 \delta}{\varepsilon \sqrt{\pi}} .\right.
$$

Lemma 1.1.2 shows that attempting to reconstruct the derivative of the mollified function is a stable problem with respect to the perturbations in the data, in the maximum norm and for a fixed radius of mollification $\varepsilon$. Under the conditions of the Lemma 1.1.1 and 1.1.2, the error estimate in this process is given by

$$
\left\|\left(J_{\varepsilon} f^{\delta}\right)^{\prime}-f^{\prime}\right\|_{\infty, I} \leq 3 \varepsilon M+\frac{2 \delta}{3 M \sqrt{\pi}}
$$

The error estimate (1.19) can be minimized by choosing $\varepsilon=\left(\frac{2 \delta}{3 M \sqrt{\pi}}\right)^{\frac{1}{2}}$. For this choice the error estimate becomes

$$
\left\|\left(J_{\varepsilon} f^{\delta}\right)^{\prime}-f^{\prime}\right\|_{\infty, I} \leq 2 \pi^{\frac{1}{4}} \sqrt{6 M} \sqrt{\delta} .
$$

This gives uniform convergence as $\delta \rightarrow 0$, that is as the quality of data improves with a rate of $\mathcal{O}(\sqrt{\delta})$. From this discussion, we observe that the new problem of computing $J_{\varepsilon} f^{\prime}$, is well-posed, depends on the parameter $\varepsilon$, and in the absence of noise in the data function is consistent with the original problem (see Lemma 1.1.1). In the presence of noise in the data function with a fixed parameter $\varepsilon>0$, The problem of finding $\left(J_{\varepsilon} f\right)^{\prime}$ is stable with respect to perturbations in the data (Lemma 1.1.2). Therefore the family $\frac{d}{d x} J_{\varepsilon}, \varepsilon>0$ is a regularizing family for the numerical differentiation problem.

\subsubsection{Compact operators}

Many ill-posed problems that arise in several applications often involve compact operators [10, 19, 27]. 
For a self-adjoint compact operator, the notion of an eigensystem plays a very important role: an eigensystem $\left(\lambda_{n}, v_{n}\right)$ consists of all non-zero eigenvalues $\lambda_{n}$ and a corresponding complete orthogonal set of eigenvectors $v_{n}$. Using this eigensystem the compact operator $K$ can be diagonalized,

$$
K x=\sum_{n=1}^{\infty} \lambda_{n}<x, v_{n}>v_{n}, \quad x \in X,
$$

where the series converges in the norm of $Y$. If the operator $K$ is not self-adjoint, then a singular system is used to replace an eigensystem.

A singular system of a compact linear operator $K$ is a countable set of tripples $\left(\sigma_{n}, v_{n}, u_{n}\right)_{n=1}^{\infty}$ with the following properties: If $K^{*}: Y \rightarrow X$ denotes the adjoint of $K$, then $\left\{\sigma_{n}^{2}\right\}_{n \in \mathcal{N}}$ are the non-zero eigenvalues of the self adjoint operator $K^{*} K$ in decreasing order with multiplicity, $\sigma_{1} \geq \sigma_{2} \geq \cdots \geq 0,\left\{v_{n}\right\}_{n \in \mathbb{N}}$ are the corresponding complete orthonormal system of eigenvectors of $K^{*} K$ and $\left\{u_{n}\right\}_{n \in \mathcal{N}}$ are the complete orthonormal system of eigenvectors of the operator $K K^{*}$. The two orthonormal systems are related as follows

$$
K v_{n}=\sigma_{n} u_{n}, K^{*} u_{n}=\sigma_{n} v_{n}
$$

Using the singular system, the the operator $K$ can then be diagonalized

$$
K x=\sum_{n=1}^{\infty} \sigma<x, v_{n}>u_{n}, \quad x \in X .
$$

The solution to the operator equation (1.7) can then be written in terms of the singular system as

$$
x=\sum_{n=1}^{\infty} \frac{1}{\sigma_{n}}<y, u_{n}>v_{n},
$$

provided that the series converges. The series converge if for all $y \in \mathcal{D}\left(K^{-1}\right)$,

$$
\sum_{n=1}^{\infty} \frac{\left|<y, u_{n}>\right|^{2}}{\sigma_{n}^{2}}<\infty .
$$

This is known as Picard's criterion for the existence of an exact solution. In practice this criterion is extremely restrictive since $y$ is generally contaminated with errors. From (1.23), it can easily be observed that solution $x$ does not depend continuously on the data hence the problem is ill-posed. This is because errors in the Fourier 
coefficients $\left\langle y, v_{n}>\right.$ are multiplied by $\frac{1}{\sigma_{n}}$ which grows to infinity as $n \rightarrow \infty$. Thus the errors in Fourier components of the data for large $n$ (high frequency errors) are strongly amplified. Also the faster the decay of the singular values, the more severe the ill-posedness, because Picard's criterion becomes more and more restrictive. This therefore makes it possible to quantify the degree of ill-posedness of the operator equation $K x=y$ in terms of singular values.

Definition 1.1.3. Degree of ill-posedness: A problem is said to be mildly ill-posed if the sequence $\left\{\sigma_{n}\right\}_{n=1}^{\infty}$ behave as $\sigma=\mathcal{O}\left(n^{-\gamma}\right)$ for some $\gamma \in \mathbb{R}^{+}$and severely ill-posed if the singular values decay exponentially, $\sigma=\mathcal{O}\left(e^{-\gamma n}\right)$.

An example of a severely ill-posed problem is the Cauchy problem for Laplace equation $[2]$.

\subsubsection{Tikhonov Regularization}

A convenient method of constructing classes of admissable regularization strategies is by filtering the singular systems. A classical method for filtering singular systems is that of Tikhonov [26]. Given a linear, bounded operator $K: X \rightarrow Y$ and $y^{\delta} \in Y$, the goal is to determine $x_{\alpha}^{\delta} \in X$ that minimizes the Tikhonov functional

$$
J_{\alpha}:=\left\|K x-y^{\delta}\right\|^{2}+\alpha^{2}\|x\|^{2}, x \in X,
$$

where $\alpha$ the regularization parameter controls the weight given to the minimization of the regularization term $\|x\|$ relative to the minimization of the residual norm $\left\|K x-y^{\delta}\right\|$. The minimization of the Tikhonov functional is therefore a compromise between minimizing the residual $\left\|K x-y^{\delta}\right\|$ and enforcing stability, that is keeping the term $\|x\|$ small.

The unique minimizer $x_{\alpha}^{\delta}$ of the Tikhonov functional is also the unique solution of the normal equation

$$
\alpha^{2} x_{\alpha}^{\delta}+K^{*} K x_{\alpha}^{\delta}=K^{*} y^{\delta}
$$

For a compact operator $K$ with a singular systems $\left(\sigma_{n}, v_{n}, u_{n}\right)$, the unique minimizer has the form

$$
x_{\alpha}^{\delta}=\sum_{n=1}^{\infty} \frac{\sigma_{n}}{\sigma_{n}^{2}+\alpha^{2}}<y^{\delta}, u_{n}>v_{n} .
$$


A comparison with equation (1.23), clearly shows how this method stabilizes computing of an approximate solution $x_{\alpha}^{\delta}$ : errors in $\left\langle y, u_{n}>\right.$ are not propagated with the factors $\frac{1}{\sigma_{n}}$ but with the factors $\frac{\sigma_{n}}{\sigma_{\alpha}^{2}+\alpha^{2}}$ which are clearly bounded as $n \rightarrow \infty$. The operators $R_{\alpha}$ are bounded with norm

$$
\left\|R_{\alpha}\right\|=\max _{n \in \mathbb{Z}^{+}}\left\{\frac{\sigma_{n}}{\sigma_{n}^{2}+\alpha^{2}}\right\} \leq \max _{\sigma>0}\left\{\frac{\sigma}{\sigma^{2}+\alpha^{2}}\right\} \leq \frac{1}{2 \alpha} .
$$

Using the error estimate (1.11) and Theorem 2.7 in [19], the error encountered when computing an approximate solution by Tikhonov regularization method can be estimated by

$$
\left\|x_{\alpha}^{\delta}-x\right\| \leq \frac{\delta}{2 \alpha}+\frac{1}{2} \alpha\|z\|
$$

where $x=K^{*} z$. The right-handside of (1.27) can be minimized by taking

$$
\alpha^{2}=\frac{\delta}{\|z\|}
$$

This gives

$$
\left\|x_{\alpha}^{\delta}-x\right\| \leq\|z\|^{\frac{1}{2}} \delta^{\frac{1}{2}}
$$

This therefore means that Tikhonov regularization with parameter choice rule for which $\left\|x_{\alpha}^{\delta}-x\right\|=\mathcal{O}\left(\delta^{\frac{1}{2}}\right)$ as $\delta \rightarrow 0$ is of optimal order for $y \in R\left(K^{*}\right)$.

The following convergence result can be found in [10].

Theorem 1.1.1. Let $x_{\alpha}^{\delta}$ be a unique minimizer of the Tikhonov functional (1.25), $y \in \mathcal{R}(K),\left\|y-y^{\delta}\right\| \leq \delta$. If $\alpha=\alpha(\delta)$ is such that

$$
\lim _{\delta \rightarrow 0} \alpha^{2}(\delta)=0 \text { and } \lim _{\delta \rightarrow 0} \frac{\delta}{\alpha(\delta)}=0
$$

then

$$
\lim _{\delta \rightarrow 0} x_{\alpha}^{\delta}=K^{-1} y
$$

Therefore for every parameter choice rule $\alpha$ satisfying the conditions of this theorem, the pair $\left(R_{\alpha}, \alpha\right)$ defines a convergent regularization method. 


\subsubsection{Parameter Choice strategies}

In this section, we discuss two parameter choice methods that are most commonly used when dealing with ill-posed problems. No regularization method is complete without the method for choosing the regularization parameter that should be used for the problem at hand. Here we consider the problem of solving the operator equation $K x=y$ but instead of the exact data $y$, only noisy data $y^{\delta}$ satisfying $\left\|y-y^{\delta}\right\| \leq \delta$ is available. Parameter choice methods are characterized into two classes depending on their assumptions about the noise level $\delta$. The two classes are as follows

1. Methods based on the knowledge or a good estimate of the noise level $\delta$.

2. Methods that do not require knowledge about the noise level but instead seek to extract information for the given right-hand side.

When reliable information about the noise level is available, then it is crucial to make use of this information and this is the basis of the Discrepancy principle, due to Morozov. The Discrepancy principle is most commonly used method of determining the level of regularization of an ill-posed problem. The regularization parameter defined via the Discrepancy principle is

$$
\alpha\left(\delta, y^{\delta}\right)=\sup \left\{\alpha>0 \mid\left\|K x_{\alpha}^{\delta}-y^{\delta}\right\| \leq \delta\right\}
$$

According to the Discrepancy principle, since $\left\|K x-y^{\delta}\right\|=\left\|y-y^{\delta}\right\|=\delta$, it does not make sense to seek for an approximate solution $x_{\alpha}^{\delta}$, with a residual $\left\|K x_{\alpha}^{\delta}-y^{\delta}\right\|<\delta$, a residual in the order of the noise level $\delta$ is the best we can ask for. When computing approximate solutions, the largest possible regularization parameter which results in a discrepancy in the order of $\delta$ should be used since smaller regularizations means less stability of numerical computations. The discrepancy principle therefore works only when there is knowledge about the level of noise in the available data.

For cases where there is no information of the level of noise in the data, noise free methods are used. The most commonly used of all parameter free methods is the Lcurve method, originally proposed by Lawson and Hanson [20]. The L-curve which is a plot of the residual norm $\left\|K x_{\alpha}^{\delta}-y^{\delta}\right\|$ verus $\left\|x_{\alpha}^{\delta}\right\|$ for different values of $\alpha$ is a 
very convenient graphical tool for analysis of ill-posed problems. The L-curve displays the compromise between the minimization of the two quantities $\left\|K x_{\alpha}^{\delta}-y^{\delta}\right\|$ and $\left\|x_{\alpha}^{\delta}\right\|$. For discrete ill-posed problems, the L-curve when plotted in log-log scale often has the characteristic L-shaped appearance with a distinct corner separating the vertical and horizontal parts of the curve. Less regularization also known as undersmoothing corresponds to the uppermost of the curve and more regularization (oversmoothing) correspond to the rightmost part of the curve.

The optimal regularization parameter that ensures a good balance between minimization of the residual norm $\left\|K x_{\alpha}^{\delta}-y^{\delta}\right\|$ and the solution norm $x_{\alpha}^{\delta}$ is that value $\alpha$ which correspond the corner of the curve $[20,15,16]$.

\subsubsection{Concrete example}

Consider the Cauchy problem for Laplace equation on a rectangle $\Omega=[0,1] \times[0,1]$;

$$
\begin{cases}\Delta u=0 & \text { in } \Omega, \\ u(x, 0)=g(x) & 0<x<1 \\ u(0, y)=u(1, y)=0 & 0<y<1 \\ u_{y}(x, 0)=0 & 0<x<1 .\end{cases}
$$

The problem of computing $f(x)=u(x, 1)$ from the given data $g(x)$ is severely illposed. To show this we solve a well-posed problem with arbitrary data $u(x, 1)=$ $f(x)$. By separation of variables the solution to the well posed problem is given by

$$
u(x, y)=\sum_{n=1}^{\infty} \frac{\cosh (n \pi y)}{\cosh (n \pi)}<f, X_{n}>X_{n},
$$

where $X_{n}=X_{n}(x)=\sqrt{2} \sin (n \pi x)$ and $<f, X_{n}>$ is defined by

$$
<f, X_{n}>=\sqrt{2} \int_{0}^{1} f(x) \sin (n \pi x) d x .
$$

Note that $\left\{X_{n}\right\}_{n=1}^{\infty}$ is the orthonormal basis of $L^{2}(0,1)$. Using the data $u(x, 0)=$ $g(x)$, we obtain

$$
g(x)=\sum_{n=1}^{\infty} \frac{1}{\cosh (n \pi)}<f(x), X_{n}>X_{n} .
$$


From (1.31), we define an operator $K: L^{2}(0,1) \rightarrow L^{2}(0,1)$, such that problem of computing $u(x, 1)=f(x)$ from the data $g(x)$ can be reformulated as the following operator equation

$$
K f(x)=g(x)
$$

where

$$
K f=\sum_{n=1}^{\infty} \frac{1}{\cosh (n \pi)}<f(x), X_{n}>X_{n} .
$$

The operator $K$ has a singular decomposition given by

$$
\left\{\sigma, v_{n}, u_{n}\right\}=\left\{\frac{1}{\cosh (n \pi)}, \sqrt{2} \sin (n \pi x), \sqrt{2} \sin (n \pi x)\right\}
$$

and is therefore a linear, self-adjoint compact operator[24]. The singular values $\frac{1}{\cosh (n \pi)}$ decay exponentially fast as $n \rightarrow \infty$ and this means computing $f(x)$ from $g(x)$ is severely ill-posed.

In order to obtain a stable approximation of the function $f(x)$, we seek to find a regularized approximation $f_{\alpha}^{\delta}(x)$ by solving the minimization problem

$$
\min _{f \in L^{2}(0,1)}\left\{\|K f-g\|^{2}+\alpha^{2}\|f\|^{2}\right\} .
$$

The unique minimizer of minimization problem is also the unique solution of the normal equation

$$
\alpha^{2} f_{\alpha}^{\delta}+K^{*} K f_{\alpha}^{\delta}=K^{*} g
$$

where $K^{*}$ is the adjoint of $K$. From (1.32) and (1.34) and the fact that $K=K^{*}$, we have the regularized solution as

$$
f_{\alpha}^{\delta}=\sum_{n=1}^{\infty} \frac{\cosh (n \pi)}{1+\alpha^{2} \cosh ^{2}(n \pi)}<g(x), X_{n}>X_{n} .
$$

\section{Numerical analysis of the example}

In this section we illustrate the ill-posedness of the Cauchy problem (1.30) and effects of Tikhonov regularization. To approximate the linear operator $K$, we discretize the problem on a uniform grid $\left(x_{i}, y_{j}\right)$ and solve equation (1.30) with a known function $f(x)$. This is a well-posed problem. We illustrate the ill-posedness of the operator 
$K$ by computing the singular value decomposition (SVD) of $K$. From the solution we obtain corresponding values of the function $g(x)$ which acts as our exact data. To simulate the effects of Tikhonov regularization numerically, we add noise of level $1 e^{-3}$ to the function $g(x)$ to simulate measured data with a certain level of noise. We then attempt to reconstruct the function $f(x)$ from the noisy data $g^{\delta}$ using Tikhonov regularization (1.26) and display the results below.
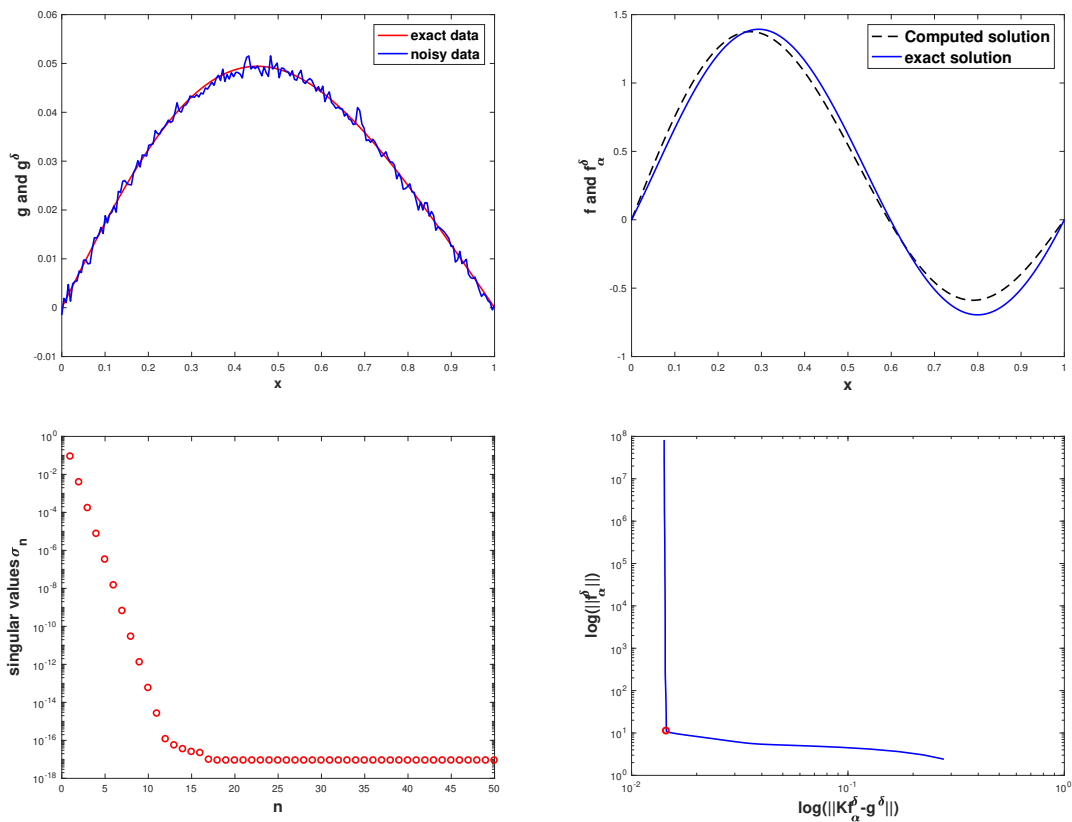

Figure 1.1:

We display the exact $g$ (solid red) and noisy data (zig zag blue) function $g^{\delta}$ (top,left), singular values of the operator $K$ (bottom, left). The L-curve (bottom,right) provides the best way to choose a regularization parameter. Particularly values around the corner (red ring) provides the best compromise between ensuring minimal residual and keeping penalty term small. We display exact value of $f$ (solid blue) and Tikhonov solution(dash black) computed using $\alpha=3.9802 e-04$ (top,right)

From Figure 1.1, We observe that the singular values decay exponentially fast implying the problem of calculting $f(x)$ from noisy data function $g^{\delta}(x)$ is severely 
ill-posed. Applying Tikhonov regularization method with a good choice of a regularization parameter $\alpha$ gives good results.

More literature about ill-posed problems, inverse problems and regularization theory can be found in [16][10][19][27]. 



\section{Summary of Papers}

\section{Paper 1}

In this paper, we consider the inverse geothermal problem where one wants to estimate the heat flux and temperature distribution below the earth's surface using measured heat flux and temperature distribution on the earth's surface. This problem is important because temperature controls many physical properties of rocks and governs a lot of geophysical process, including the generation of magmas and deformation style of rocks. Thus, the knowledge of the thermal structure of the earth's subsurface is important for understanding geophysical observations and dynamical processes in the Lithosphere. Mathematically this problem is formulated as a Cauchy problem for a non-linear stationary heat equation. The problem is severely ill-posed in the sense that the solution (if it exists) does not depend continuously on that measured data. First we consider the direct problem;

$$
\begin{array}{lr}
\left(k T_{x}\right)_{x}+\left(k T_{z}\right)_{z}+A_{p}=0 & 0<x<L_{x}, 0<z<L_{z} \\
T_{x}(x 0, z)=T_{x}\left(L_{x}, z\right)=0 & 0<z<L_{z}, \\
T(x, 0)=T_{0}(x) & 0<x<L_{x} \\
k T_{z}\left(x, L_{z}\right)=Q_{m} & 0<x<L_{x}
\end{array}
$$

with artificial data $Q_{m}$. Since the problem is non-linear, we discretize the problem using finite differences and solve the problem using an iterative procedure. The convergence of the iterative procedure is very fast since the stopping criterion is achieved with in few iterations. Using the well-posed problem (2.1), we define an operator equation

$$
K Q_{m}=k T_{z}(x, 0)=Q_{0} .
$$

When $k$ and $A_{p}$ are independent of temperature, the problem is linear. Theoretical and numerical analysis of the singular system of the linear problem shows the 
problem is severely ill-posed.

The goal in this paper is solve the non-linear problem and this is achieved by solving a sequence of linear problems using Tikhonov regularization method. We demonstrate that Tikhonov regularization can be implemented efficiently for the non-linear operator equation 2.2. Numerical results for two dimensional domains shows that the algorithm works efficiently and the solution to inverse problem can be obtained accurately with a realistic noise in the measured surface data.

\section{Paper 2}

In this paper, we investigate the solvability of a Cauchy problem for the non-linear elliptic equation. This problem appears in several applications, including the inverse geothermal problem; where the heat flux and temperature distribution in the subsurface are calculated from temperature and flux measurements on the surface. We reformulate the Cauchy problem as a non-linear operator equation. The operator equation is defined in terms of solving a boundary value problem

$$
\begin{array}{lr}
-\nabla \cdot(k(x, T) \nabla T)+b(x, T)=0, & \text { in } \Omega \\
\left.T\right|_{\Gamma_{0}}=\phi_{0} & \text { on } \Gamma_{0}, \\
\left.\vec{n} \cdot k \nabla T\right|_{\Gamma_{1}}=\psi_{1} & \text { on } \Gamma_{1},
\end{array}
$$

where $T$ is temperature, $k(x, T)$ is a thermalconductivity and $b(x, T)$ is a source term. By making some possible optimal assumptions on the coefficients of the boundary value problem, we demostrate existence and uniqueness of a solution to the boundary value problem (2.3) using an iterative procedure. An important step in this analysis is a change of variables which removes non-linearity from the leading term of the equation and allows to perform analysis under close to minimal assumptions on the coefficient of the problem.

Using appropriate function spaces we obtain estimates of the solution. We make use of these estimates to study the properties of the operator equation. The operator equation is shown to be well-defined and bounded. A numerical example is given to illustrate the convergence speed of the iterative procedure used to show existence of a unique solution. 


\section{References}

[1] G Alessandrini, L Del Piero, and L Rondi. Stable determination of corrosion by a single electrostatic boundary measurement. Inverse problems, 19(4):973-984, 2003.

[2] Fredrik Berntsson and Eldén Lars. Numerical solution of a cauchy problem for the laplace equation. Inverse Problems, 17(4):839, 2001.

[3] Fioralba Cakoni and David L Colton. A qualitative approach to inverse scattering theory. Springer, 2014.

[4] V Cermak and L Bodri. Temperature structure of the lithosphere based on $2 \mathrm{~d}$ temperature modeling applied to central and eastern europe. Thermal modeling in sedimentary basins. Editions Technip, Paris, pages 7-32, 1986.

[5] Lin Chen, Fredrik Berntsson, Zhongjie Zhang, Peng Wang, Jing Wu, and Tao $\mathrm{Xu}$. Seismically constrained thermo-rheological structure of the eastern tibetan margin: Implication for lithospheric delamination. Tectonophysics, 627(0):122$134,2014$.

[6] Murio Diego A. The mollification method and the numerical solution of ill-posed problems. Wiley, Newyork, 1993.

[7] Lars Eldén. Numerical solution of the sideways heat equation by difference approximation in time. Inverse Problems, 11(4):913, 1995.

[8] Lars Eldén, Fredrik Berntsson, and Teresa Reginska. Wavelet and fourier methods for solving the sideways heat equation. SIAM Journal on Scientific Computing, 21(6):2187-2205, 2000.

[9] Lars Eldén and Valeria Simoncini. A numerical solution of a cauchy problem for an elliptic equation by krylov subspaces. Inverse Problems, 25(6):065002, 2009.

[10] Heinz Werner Engl, Martin Hanke, and Andreas Neubauer. Regularization of inverse problems, volume 375. Springer Science \& Business Media, 1996. 
[11] Georgios Fotopoulos and Valery Serov. Inverse fixed energy scattering problem for the two-dimensional nonlinear schrödinger operator. Inverse Problems in Science and Engineering, 24(4):692-710, 2016.

[12] Matthias Gehre, Tobias Kluth, Antti Lipponen, Bangti Jin, Aku Seppänen, Jari P Kaipio, and Peter Maass. Sparsity reconstruction in electrical impedance tomography: an experimental evaluation. Journal of Computational and Applied Mathematics, 236(8):2126-2136, 2012.

[13] Gerardo González, Ville Kolehmainen, and Aku Seppänen. Isotropic and anisotropic total variation regularization in electrical impedance tomography. Computers $\&$ Mathematics with Applications, 2017.

[14] Jacques Hadamard. Lectures on Cauchy's problem in linear partial differential equations. Dover Publications, New York, 1953.

[15] Per Christian Hansen. Rank-deficient and discrete ill-posed problems. JSTOR, 1996.

[16] Per Christian Hansen. Rank-deficient and discrete ill-posed problems: numerical aspects of linear inversion. SIAM, 1998.

[17] Gabriele Inglese. An inverse problem in corrosion detection. Inverse problems, 13(4):977, 1997.

[18] Peter G Kaup, Fadil Santosa, and Michael Vogelius. Method for imaging corrosion damage in thin plates from electrostatic data. Inverse problems, 12(3):279, 1996.

[19] Andreas Kirsch. An introduction to the mathematical theory of inverse problems, volume 120. Springer Science \& Business Media, 2011.

[20] Charles L Lawson and Richard J Hanson. Solving least squares problems, volume 161. SIAM, 1974.

[21] HH Qin and T Wei. Two regularization methods for the cauchy problems of the helmholtz equation. Applied Mathematical Modelling, 34(4):947-967, 2010. 
[22] Valery Serov, Markus Harju, and Georgios Fotopoulos. Direct and inverse scattering for nonlinear schrödinger equation in 2d. Journal of Mathematical Physics, 53(12):123522, 2012.

[23] D Stromeyer. Downward continuation of heat flow data by means of the least squares method. Tectonophysics, 103(1):55-66, 1984.

[24] Ulrich Tautenhahn. Optimal stable solution of cauchy problems for elliptic equations. journal $f$ " $u$ r Analysis and its applications, 15(4):961-984, 1996.

[25] S Thiagarajan, DV Ramana, and SN Rai. Seismically constrained twodimensional crustal thermal structure of the cambay basin. Journal of Earth System Science, 110(1):1-8, 2001.

[26] Andrey Tikhonov. Solution of incorrectly formulated problems and the regularization method. In Soviet Math. Dokl., volume 5, pages 1035-1038, 1963.

[27] Curtis R Vogel. Computational methods for inverse problems. SIAM, 2002. 


\section{Papers}

The papers associated with this thesis have been removed for copyright reasons. For more details about these see:

http:// urn.kb.se/ resolve?urn=urn:nbn:se:liu:diva-143031 